\title{
NOTAS SOBRE ECOLOGÍA Y DISTRIBUCIÓN DEL GÉNERO LEPANTHES (ORCHIDACEAE) EN CUBA, CON UNA LISTA ACTUALIZADA Y REVISADA
}

\author{
Juan A. Llamacho Olmo \\ Instituto de Ecología y Sistemática (IES). Carretera de Varona Km 3 1/2 \\ Capdevila, Boyeros, Ciudad de la Habana. Cuba. \\ botanica.ies@ama.cu / llamacho@yahoo.com
}

\begin{abstract}
Notes about ecology and distribution of the genus Lepanthes in Cuba are given. The paper includes a list of all the species recorded for the island, and the location of their types in herbaria.

RESUMEN. Se ofrecen notas sobre la ecología y la distribución del género Lepanthes en Cuba, con una lista de las especies registradas en la isla y de los herbarios donde se encuentran depositados los tipos.
\end{abstract}

Palabras ClaVe / KeY Words: Orchidaceae, Lepanthes, ecología, Cuba.

El género Lepanthes fue descrito por Swartz en 1799 a partir de plantas recolectadas en Jamaica, que originalmente habían sido incluidas en el género Epidendrum. Para algunos autores, como Salazar \& Soto (1996), el nombre genérico se origina a partir de los términos griegos lepis (envoltura) y anthos (flor), haciendo tal vez referencia al hábitat epífito de estas plantas que viven sobre la corteza de los árboles, vista como una envoltura; otros autores lo interpretan como "flores pequeñas con aspecto de escama", porque el término lepis significa también escama (Luer 1986).

Según la clasificación de Dressler (1981), el género se ubica en la subfamilia Epidendroideae, tribu Epidendreae, subtribu Pleurothallidinae. Luer (1986) realiza una división taxonómica y considera cuatro subgéneros: Lepanthes, Brachycladium, Marsipianthes y Draconanthes; el primero es el único presente en Cuba. Los tres restantes corresponden a especies de la región andina, que se considera el centro de diversificación de este género (Luer 1986).

Lepanthes es un género neotropical que se distribuye desde México hasta Bolivia y el norte de Brasil; también está presente en el arco antillano. Posee alrededor de 800 especies descritas, cifra que puede aumentar debido a que crecen en áreas de difícil acceso.

La primera recolecta del género Lepanthes en Cuba fue realizada por Charles Wrigth en el siglo XIX, en la zona oriental de la isla. Las especies recolectadas por Wright fueron estudiadas y descritas por Lindley en 1858 (Howard 1988).

Wright recolectó nueve especies, pero varias no fueron tomadas en cuenta por Lindley por encontrarse hasta tres especies diferentes en un solo ejemplar. Entre las especies recolectadas por Wright y posteriormente descritas por Lindley se encuentran L. blepharophylla (Wright 1508), de la zona de Monte Verde, Guantánamo; L. dorsalis (Wright 662) y L. trichodactyla (Wright 661) de la Sierra Maestra.

Reichenbach fil. (1865, en Flora 48) estudió los especímenes de Wright y agregó dos nuevas especies: L. wrightii (Wright 3340) y L. mandibularis (Wright 1342), ambas del macizo montañoso Moa-Baracoa.

A principios del siglo XX, el sueco E.L. Ekman exploró la región oriental del país; recolectó cinco nuevas especies de Lepanthes, descritas por Schlechter en 1923. Resulta significativo que algunas de ellas ya habían sido recolectadas con anterioridad por Wright, pero aún no habían sido descritas. Entre las especies descritas como nuevas se encuentra $L$. melanocaulon (Ekman 3361) de la Sierra de Nipe. Las especies restantes fueron recolectadas en la escalada de Ekman al Pico Turquino: L. blepharantha (Ekman 5491), L. ekmanii (Ekman 5409), L. pergracilis (Ekman 5490) y L. turquinoensis (Ekman 5438).

Todas las especies encontradas en Cuba hasta ese momento fueron registradas por Julián Acuña en el Catálogo de Orquídeas Cubanas, publicado en 1938 (Acuña 1938). Posteriormente, se realizó una revisión del género en la obra Flora de Cuba, en la que 
aparece un total de 21 especies (León 1946).

En 1973 Hespenheide publicó una revisión del género en Cuba, en la que recopiló 18 especies. Este trabajo se basó estrictamente en el estudio de los ejemplares depositados en herbarios, ya que el autor reconoció que nunca había visitado el país. En ese artículo describe seis especies nuevas y realiza una nueva combinación: L. acuñae, L. blepharophylla, $L$. cubensis, L. dressleri, L. grisebachiana, L. occidentalis y L. obliquiloba (Hespenheide 1973).

En 1988 Helga Dietrich describió L. silvae, recolectada en la región de Moa, en el norte de Oriente (Dietrich 1988).

Una expedición formada por renombrados especialistas en la familia Orchidaceae recorrió el norte de la región oriental de Cuba en 1997. Producto de la misma, C. Luer describió seis nuevas especies: $L$. comadresina, L. diaziae, L. llamachoi, L. minimamundana, L. palpelabris y L. woodfredensis (Luer 1998). Con posterioridad a esta publicación, Luer continuó trabajando con materiales recolectados en varias regiones de la isla y ha publicado las siguientes especies: L. aubryi, L. cyrillicola, $L$. decoris y $L$. nana, de la región del Pico Turquino, y L. martae de la región de Cuchillas de Moa-Baracoa (Luer 1999, 2001, 2002).

Distribución. El género Lepanthes está restringido únicamente a las montañas de la isla y presenta la mayor diversidad en el macizo montañoso NipeSagua-Baracoa con un total de 16 especies; le sigue la Sierra Maestra, con 13, el Macizo Guamuhaya, con tres, y la Cordillera de Guaniguanico, con dos. Es muy probable que en todas las regiones montañosas de Cuba existan especies nuevas por descubrir.

Este género está adaptado a condiciones ecológicas muy especificas, pues requiere de una humedad relativa muy alta y preferentemente regiones neblinosas. En la Sierra Maestra sólo se encuentra por encima de 1000 metros de altitud, debido a que a esta altura predominan dichas condiciones. En el Macizo NipeSagua-Baracoa comienzan a aparecer en las laderas con exposición norte, a partir de 300 metros de altitud, generalmente en las zonas de condensación de la humedad de los vientos procedentes del mar. Los sistemas montañosos del centro y del occidente de la isla presentan las mismas características.

Varias especies tienen una distribución muy restringida y sólo se conocen de la localidad tipo; de otras únicamente se conocen una o dos poblaciones. Esta característica atenta contra la conservación de muchos de estos taxa. Particularmente crítico es el caso de L. comadresina, que fue recolectada por única vez en Arroyo Las Comadres en Moa, en 1997, donde se encontró creciendo en las márgenes del arroyo, y en posteriores expediciones a la zona en 2000 y 2002 no fue hallada. Presumiblemente, la población se extinguió durante una severa sequía que sufrió la zona en 1999; no obstante, aún no se ha podido comprobar si se trata de una extinción total de la especie, o si ésta podrá recolonizar el área. Fluctuaciones poblacionales también han sido observadas en especies mexicanas que sufren extinciones locales.

Hábitat. Las plantas se caracterizan por ser epifitas o litófitas, aunque muchas de ellas prefieren indistintamente estos dos sustratos, pero hay otras que son estrictamente epífitas o litófitas.

Las formaciones vegetales que mayor número de Lepanthes albergan son el bosque nublado, en la región del Turquino, y los bosques pluviales de MoaBaracoa. Incluso en estos bosques las poblaciones son más densas en las áreas de mayor condensación. Muchas de estas especies se encuentran vinculadas a cursos de agua, sobre todo en la región nororiental de la isla. A pesar de lo antes descrito, en el bosque pluvial de Monte Iberia, en el macizo Moa-Baracoa, que es una zona de alta humedad y numerosas precipitaciones, prácticamente no existen especies de Lepanthes, lo que podría deberse a la competencia por el sustrato, dado que en los troncos de los árboles predominan absolutamente musgos y helechos.

Fenología. Todas las especies del género florecen durante todo el año; sólo dejan de hacerlo ante la ocurrencia de períodos de sequía que provocan estrés hídrico. Esta aseveración ha sido corroborada con observaciones independientes en Sierra de Nipe, Sierra de Moa y Pico Turquino. Cuando pasan varios días sin lluvia o rocío, las flores se cierran y se pueden mantener en esas condiciones por 3 o 4 días, para volver a abrirse con el aumento de la humedad. Cuando las condiciones secas se mantienen por más de 10 días las flores mueren, pero quedan las yemas latentes, que posibilitan comenzar la floración del 
mismo escapo cuando cambian las condiciones ambientales.

Una de las características más singulares de este grupo es la coexistencia y la floración simultánea de varias especies en un mismo sustrato. Por ejemplo, en una sola piedra se han encontrado juntas $L$. fractiflexa, L. turquinoensis, L. ekmanii y L. trichodactyla. Resulta una incógnita cómo logran el aislamiento reproductivo pese a que todas florecen al mismo tiempo; es probable que esté garantizado por el mecanismo de polinización.

Respecto a los polinizadores del género prácticamente no hay resultados de investigación. Sólo se ha identificado el mosquito de los hongos Bradysia sp. (Sciaridae) como polinizador de L. glicensteinii Luer, de Costa Rica (Blanco \& Barboza 2001).

\section{LISTA DE ESPECIES CUBANAS CON TIPOS Y DISTRIBUCIÓN GEOGRÁFICA}

En Cuba se reconoce en la actualidad un total de 29 especies de Lepanthes.

1. Lepanthes acuñae Hespenh., Brittonia 25: 263. 1973.

TIPO: CuBA. Entre Pico Turquino y La Bayamesa, C. Morton \& J. Acuña 3675 (Holotipo: US!).

DISTRIBUCIÓN: Pico Turquino, Sierra Maestra.

2. Lepanthes aubryi Luer \& P. Jesup, Selbyana 23(1): 4. 2002.

TIPO: CuBA. Santiago de Cuba, Sierra Maestra, Pico Turquino, Y. Aubry, dic. 2000, cultivado en Bristol, CT, P. Jesup 8 (Holotipo: MO).

DisTRIBUCIÓN: Pico Turquino, Sierra Maestra.

3. Lepanthes blepharantha Schltr., Urban Symb. Antill. 9: 61. 1923.

TIPO: CuBA. Ladera $\mathrm{N}$ del Pico Turquino, $E$. Ekman 5491 (Holotipo: S).

DisTRIBUCIÓN: Pico Turquino, Sierra Maestra.

4. Lepanthes blepharophylla (Griseb.) Hespenh., Brittonia 25: 260. 1973.

Basiónimo: Pleurothallis blepharophylla Griseb., Cat. Pl. Cub. 260. 1866.

TIPO: CuBA. Sierra de Toa, cerca de Monte Verde, C. Wright 1508 (Holotipo: GOET!; isotipo: AMES).

DISTRIBUCIÓN: Conocida sólo de la localidad tipo.
5. Lepanthes chrysostigma Lindl., Ann. Mag. Nat. Hist. III 1: 329. 1858

TIPO: CubA. Sierra de Toa, Monte Verde, $C$. Wright s.n. (Holotipo: K!).

DISTRIBUCIÓN: Macizo Moa-Baracoa.

6. Lepanthes comadresina Luer, Lindleyana 13(3): 138. 1998.

TIPO: CuBA. Holguín: Moa, camino a la Melba, Arroyo las Comadres, 350 m, 29 nov. 1997, C. Luer, J. Luer, M. Díaz, J. Llamacho, J. Ackerman, K. \& R. Dressler 18650 (Holotipo: HAJB; isotipo: MO).

Distribución: Arroyo Las Comadres, La Melba, Moa. Holguín.

7. Lepanthes cubensis Hespenh., Brittonia 25: 269. 1973.

TIPO: Cuba. Sierra de Toa, Monte Verde, $C$. Wright 1512 (Holotipo: PH!; isotipos: AMES, BR!, $\mathrm{K}$ !, NY, MO!,W!)

DISTRIBUCIÓN: Macizo Moa-Baracoa.

8. Lepanthes cyrillicola Luer \& Llamacho, Selbyana 22(2): 104. 2001.

TIPO: CUBA. Pico Turquino. Sierra Maestra, $J$. Llamacho 0014 (Holotipo: HAC; isotipo: MO).

DISTRIBUCIÓN: Pico Turquino, Sierra Maestra.

9. Lepanthes decoris Luer \& Llamacho, Selbyana 22(2): 106. 2001.

TIPO: CuBA. Paso del mono, Pico Turquino, Sierra Maestra. 5 jun. 1999, J. Llamacho 0010a (Holotipo: HAC; isotipo: $\mathrm{MO}$ ).

DistRIBUCIÓN: Pico Turquino, Sierra Maestra

10. Lepanthes diaziae Luer, Lindleyana 13(3): 138. 1998.

TIPO: CuBA. Holguín, Mayarí, Sierra de Nipe, epífito en bosque pluvial, Cayo las Mujeres, $650 \mathrm{~m}, 25$ nov. 1997, C. Luer, J. Luer, M. Díaz, J. Llamacho, J. Ackerman, K. \& R. Dressler 18622 (Holotipo: HAJB; isotipos: MO, UPRRP).

DisTRIBUCIÓN: Sierra de Nipe y Sierra Cristal.

11. Lepanthes dorsalis Lindl., Ann. Mag. Nat. Hist. III 1: 329. 1858.

TIPO: CubA. Cresta de Nima-Nima, Sierra Maestra, C. Wright 662 (Holotipo: K!)

DISTRIBUCIÓN: Sierra Maestra y Sierra de Nipe. 
12. Lepanthes dressleri Hespenh., Brittonia 25: 268. 1973.

TIPO: CubA. Pico Sombrero, NE del Naranjo, Trinidad, R.L.Dressler 1335 (Holotipo: US2399322!)

Distribución: Sierra del Escambray. Sierra del Rosario y Sierra del Infierno.

13. Lepanthes ekmanii Schltr., Urban Symb. Antill. 9: 62. 1923.

TIPO: CuBA. Pico Turquino, Sierra Maestra, E. Ekman 5409 (Holotipo: S)

DisTRIBUCIÓN: Pico Turquino, Sierra Maestra.

14. Lepanthes fractiflexa Ames \& C. Schweinf., Sched. Orch. 10: 42. 1930.

TIPO: CuBA. Cueva del aura, Pico Turquino, Bucker 30 (Holotipo: NY; isotipo: AMES)

DISTRIBUCIÓN: Pico Turquino, Sierra Maestra.

15. Lepanthes fulva Lindl., Ann. Mag. Nat. Hist. III 1: 329.1858.

TIPO: CubA. Sierra del Toa, cerca de Monte Verde, C. Wright s.n. (Holotipo: K!).

DISTRIBUCIÓN: Macizo Moa-Baracoa, Sierra de Nipe y Sierra Maestra.

16. Lepanthes grisebachiana Hespenh., Brittonia 25: 272. 1973.

TIPO: CubA. Sierra del Toa, cerca de Monte Verde, C. Wright 1510 (Holotipo: PH!; isotipos: BR, $\mathrm{MO}$ !, W!)

DisTRIBUCIÓN: Macizo Moa-Baracoa, Sierra Cristal.

17. Lepanthes Ilamachoi Luer, Lindleyana 13(3): 138. 1998.

TIPO: CuBA. Holguín: Moa, camino a la Melba, Arroyo las Comadres, 350 m, 29 nov. 1997, C. Luer, J. Luer, M. Díaz, J. Llamacho, J. Ackerman, K. \& R. Dressler 18651 (Holotipo: HAJB; isotipo: MO).

DISTRIBUCIÓN: Sierra de Moa, Oriente.

18. Lepanthes martae Luer, Selbyana 22(2): 109. 2001.

TIPO: CuBA. Datos de recolecta no disponibles, 1999, M.A. Díaz 10 (Holotipo: HAC; isotipo: MO).

DistRIBUCIÓN: Macizo Montañoso Toa-Baracoa.

Nota: En la publicación original no existen datos de la colección, pero la especie fue recolectada en las montañas de Guantánamo (M.A. Díaz, com. pers. 2003).
19. Lepanthes melanocaulon Schltr., Urban Symb. Antill. 9: 64. 1923.

TIPO: CUBA. Río Piloto, Sierra de Nipe, E. Ekman 3361 (Holotipo: S; isotipos: Herb. Garay!, NY)

DISTRIBUCIÓN: Macizo Nipe-Sagua-Baracoa.

20. Lepanthes nana Luer \& P. Jesup, Selbyana 23(1): 17. 2002.

TIPO: CubA. Santiago de Cuba: Sierra Maestra, Pico de Santiago, 1200 m, Y. Aubry, mayo 2000, cultivado en Bristol, P. Jesup s.n. (Holotipo: MO).

DISTRIBUCIÓN: Pico Turquino, Sierra Maestra.

Nota: El topónimo Pico de Santiago no existe. La especie fue recolectada en una zona de la Sierra Maestra, perteneciente a la provincia de Santiago de Cuba.

21. Lepanthes occidentalis Hespenh., Brittonia 25: 276. 1973.

TIPO: CuBA. $12 \mathrm{~km}$ al $\mathrm{S}$ de Manicaragua, Las Villas, R.L. Dressler 1294 (Holotipo: US!; isotipo: $\mathrm{MO}$ !)

DISTRIBUCIÓN: Sierra del Escambray.

22. Lepanthes obliquiloba Hespenh., Brittonia 25: 273. 1973.

TIPO: CuBA. Las Villas, $3 \mathrm{~km}$ NE del Naranjo, Sierra de Trinidad. R.L. Dressler 1333 (Holotipo: US!, isotipo: MO!)

DISTRIBUCIÓN: Sierra del Escambray y Sierra del Rosario.

23. Lepanthes palpelabris Luer, Lindleyana 13(3): 138. 1998.

TIPO: CUBA. Holguín: Moa, camino al Toldo, Alto de la Calinga, 950 m, 30 nov. 1997, C. Luer, J. Luer, M. Díaz, J. Llamacho, J. Ackerman, K. \& R. Dressler 18658 (Holotipo: HAJB; isotipos: MO, UPRRP).

DISTRIBUCIÓN: Meseta del Toldo, Moa, Holguín.

24. Lepanthes pergracilis Schltr., Urban Symb. Antill. 9: 65. 1923.

TIPO: CuBA. Ladera N del Pico Turquino, $1950 \mathrm{~m}$, E. Ekman 5490, pro parte (Holotipo: S; isotipo: Herb. Garay!).

Distribución: Pico Turquino y Pico Bayamesa, Sierra Maestra.

25. Lepanthes silvae H. Dietr., Wiss. Zeitschr. Friedrich-Schiller-Univ. Jena 37(1): 157. 1988. TIPO: CuBA. Sierra de Moa. Álvarez et al. (HAJB). 
DistribuCiÓN: Macizo Montañoso Sagua-Baracoa.

26. Lepanthes trichodactyla Lindl., Ann. Mag. Nat. Hist. III 1: 329. 1858.

TIPO: CubA. Cresta de Nima-Nima, Sierra Maestra, C. Wright 661 (Holotipo: K-L!; isotipos: AMES, pro parte, Herb. Garay, K-Herb. Hooker!, PH!, W!).

DistribuCiÓN: Sierra Maestra y Macizo Nipe-SaguaBaracoa.

27. Lepanthes turquinoensis Schltr., Urban Symb. Antill. 9: 65. 1923.

TIPO: CubA. Sierra Maestra,Ladera N del Pico Turquino, 1800 m, E. Ekman 5438 (Holotipo: S).

DisTRIBUCIÓN: Pico Turquino, Sierra Maestra.

28. Lepanthes woodfredensis Luer, Lindleyana 13(3): 138. 1998.

TIPO: CuBA. Holguín: Mayarí, Sierra de Nipe, epífito en bosque pluvial, Cayo Mujeres, $750 \mathrm{~m}, 25$ nov. 1997, C.A. Luer, J. Luer, M. Díaz, J. Llamacho, J. Ackerman \& R.L. Dressler 18623 (Holotipo: HAJB; isotipo: MO).

DISTRIBUCIÓN: Sierra de Nipe, Oriente.

29. Lepanthes wrightii Rchb.f., Flora 48: 275. 1865.

TIPO: CuBA. Sin localidad, C. Wright 3340

(Holotipo: W!, dibujo; isotipos: AMES, BR!, K!).

DISTRIBUCIÓN: Macizo Moa-Baracoa.

RELACIÓN DE SINÓNIMOS O REGISTROS ERRÓNEOS DE LEPANTHES EN CUBA (en negrita el nombre aceptado)

Lepanthes brevipetala Fawc. \& Rendle = Lepanthopsis melanantha (Rchb.f.) Ames

Lepanthes ciliata Lindl. ex Griseb. = Lepanthes blepharophylla (Griseb.) Hespenh.

Lepanthes leonii C. Schweinf. ex León = Lepanthopsis microlepanthes (Griseb.) Ames

Lepanthes lindmaniana Schltr. = L. fulva Lindl.

Lepanthes loddigesiana Rchb.f. = especie de Jamaica; confundida con $\boldsymbol{L}$. dorsalis Lindl.

Lepanthes longicruris Schltr. $=$ L. trichodactyla Lindl,

Lepanthes mandibularis Rchb.f. $=\boldsymbol{L}$. chrysostigma Lindl.
Lepanthes minima-mundana Luer $=$ L. silvae $\mathrm{H}$. Dietr.

Lepanthes ovalis (Sw.) Fawc. \& Rendle = especie de Jamaica; confundida con L. melanocaulon Schltr.

Lepanthes ovata Ames \& C. Schweinf.= L. ekmanii Schltr.

Lepanthes pristidis Rchb.f. $=$ especie de México, considerada sinónimo de $\boldsymbol{L}$. disticha (A. Rich \& Galeotti) Garay \& R.E. Schult.; los registros de Cuba corresponden a Lepanthes cubensis Hespenh. Acuña (1938) menciona esta especie de la Sierra Nipe, sin hacer referencia a ningún ejemplar de herbario. No se ha encontrado material de herbario ni poblaciones en la región, por lo que la distribución queda confinada al macizo MoaBaracoa.

Lepanthes tridentata (Sw.) Sw. = especie de Jamaica; confundida con L. grisebachiana Hespenh.

Lepanthopsis blepharophylla (Griseb.) Garay = Lepanthes blepharophylla (Griseb.) Hespenh.

Pleurothallis blepharophylla Griseb. = Lepanthes blepharophylla (Griseb.) Hespenh.

Agradecimientos. Al Dr. Carlyle Luer por todas sus enseñanzas y contribuciones al estudio del género Lepanthes en Cuba. Este trabajo se realizó bajo el auspicio del proyecto del Programa Ramal: 2043 de Colecciones Biológicas.

\section{LITERATURA CITADA}

Acuña Galé, J. 1938. Catálogo Descriptivo de las Orquídeas Cubanas. Est. Exp. Agron. Santiago de las Vegas. No. 60.

Blanco, M. \& G. Barboza. 2001. Polinización de Lepanthes: un nuevo caso de pseudocopulación en las orquídeas. Memorias 2do Seminario Mesoamericano de Orquídeología y Conservación. San José, Costa Rica.

Dietrich, H. 1988. Orchidaceae cubanae novae VI. Lepanthes silvae H. Dietrich. Wiss. Zeitschr. FrierichSchiller-Univ. Jena 37(1): 157.

Dressler, R.L. 1981. The orchids: natural history and classification. Cambridge, Mass., Harvard Univ. Press.

Hespenheide, H.A. 1973. A revision of the West Indian species of Lepanthes (Orchidaceae). III. Cuba. Brittonia 25: 257-283.

Howard, R.A. 1988. Charles Wright in Cuba, 1856-1867. Meckler Corporation. United States. 
León, H. (Sauget). 1946. Flora de Cuba, Vol. 1. Contr. Ocas. Mus. Hist. Nat. Col. La Salle. No. 8.

Luer, C.A. 1986. Systematics of the Pleurothallidinae (Orchidaceae). Icon. Pleuroth. I. Missouri Botanical Garden.

Luer, C.A. 1998. New species of orchids from Cuba. Lindleyana 13(3): 138-147.

Luer, C.A. 1999. New species of Pleurothallis from Cuba and Hispaniola. Lindleyana 14(2): 106-121.

Luer, C.A. 2001. Miscellaneous New Species in the Pleurothallidinae (Orchidaceae). Selbyana 22(2): 103-127.

Luer, C.A. 2002. Miscellaneous New Species in the Pleurothallidinae (Orchidaceae). Selbyana 23(1): 1-45.

Salazar, G.A. \& M.A. Soto. 1996. El género Lepanthes Sw. en México. Asociación Mexicana de Orquideología, A.C. 